\title{
Effectiveness of daily outpatient alcohol detoxification by an Irish public psychiatric hospital - A pilot study
}

\author{
Vincent Agyapong, Jackie Benbow, Roy Browne
}

Ir J Psych Med 2007; 24(1): 23-26

\section{Abstract}

Objective: To assess the effectiveness of daily outpatient alcohol detoxification in an Irish public psychiatric hospital.

Method: The outpatient records of patients presenting to the Assessment Unit of St Brendan's Hospital in one year (August 2004-July 2005) with symptoms of Alcohol Dependence Syndrome (ADS) and commencing daily outpatient detoxification were examined retrospectively for parameters relevant to the objectives of the study. The results were compiled and analysed using descriptive statistics.

Results: Forty patients underwent outpatient alcohol detoxification in one year and complete records were available for 32 patients (80\%). Twenty patients had fixed addresses in the hospital catchment areas, eight patients had no fixed addresses and the remaining four patients had addresses outside the catchment areas. Seven patients $(22 \%)$ presented with a co-morbid psychiatric condition including depression (four patients), anxiety disorder (two patients) and personality disorder (one patient). All seven patients were known to psychiatric sector services. Of the 32 patients commencing detoxification, 28 patients (87.5\%) attended on the second day whilst 22 patients (69\%) attended their third day's appointment. Only 17 patients (53\%) completed the outpatient detoxification. Thirteen patients $(40.6 \%)$ received at least two outpatient detoxifications during the year; of whom seven patients $(58 \%)$ received their second detoxification within two months of the first one. The record of 20 patients (62.5\%) showed that they had received advice regarding selfreferral to counselling services.

Conclusion: A high proportion of patients (47\%) presenting with symptoms of ADS did not complete daily outpatient detoxifications. A high proportion of all patients (40.6\%) also underwent multiple outpatient detoxifications during the year. It is possible that the separation between

\footnotetext{
*Vincent Agyapong, BSc, MBChB, DCP, Senior House Officer in Psychiatry, RCSI Rotational Training Scheme in Psychiatry Jackie Benbow, MBChB, MRCPsych, Consultant in General Adult Psychiatry and Clinical Director, Department of Psychiatry, Connolly Hospital, Blanchardstown, Dublin 15, Ireland.

Roy Browne, LRCP+SI, MRCPsych, MD, Consultant in General Adult Psychiatry, St Brendan's Hospital, Dublin 7, Ireland.

${ }^{*}$ Correspondence

SUBMITTED: JUNE 12, 2006. ACCEPTED: NOVEMBER 22, 2006.
}

alcohol detoxification and alcohol counselling services in Ireland contributed to these disappointing results.

Key Words: Alcohol; Alcohol Dependence Syndrome (ADS); outpatient detoxification; alcohol counselling services.

\section{Introduction}

St Brendan's Hospital is a psychiatric hospital in Dublin established over 200 years ago. It provides acute admission beds for the catchment areas of Cabra and Finglas with a combined population of about 84,000 . It also provides psychiatric services for the homeless population in the central Dublin area and also special care/intensive care services for the greater Dublin area with a population of 1.3 million. St Brendan's Hospital also frequently provides services to people living outside the catchment areas. These people are often referred to as non-district patients. In the 1960s the hospital had an inpatient population of over 2,000 patients. This number has progressively declined over time to a current inpatient population of 120 residents. With the decision by the Health Service Executive to close down the hospital to new admissions in the very near future, this number is expected to fall further.

The hospital treats a wide variety of cases ranging from schizophrenia to depression, personality disorder and neurotic disorders, but of significance to this study is the large number patients (40 patients in one year) treated for ADS in the hospital as outpatients.

According to the Second Report of the Strategic Task Force on Alcohol (STFA), ${ }^{1}$ Ireland's alcohol related problems continue to increase and in 2003 cost the society in excess of $€ 2.65$ billion. The report states that the Irish are amongst the highest consumers of alcohol in Europe, with a consumption rate of 13.5 l of pure alcohol per adult in 2003 . It indicates that alcohol related mortality increased in line with the increase in alcohol consumption between 1992 and 2002 , in particular alcohol specific chronic conditions, eg. dependency, abuse and psychosis (+61\%) and acute conditions, eg. alcohol poisoning (+90\%).

Globally, the lifetime prevalence of alcohol problems is thought to be between $13.7 \%$ and $25.5 \%$, indicating that these problems are common in ambulatory patients. ${ }^{2}$ In the US for example, more than 700,000 people receive treatment for alcohol dependence on any given day. ${ }^{3}$

Detoxification from alcohol can be undertaken in ambulatory settings with patients who are alcohol-dependent and show signs of mild to moderate withdrawal when they are not drinking. An appropriate candidate for outpatient detoxifica- 
tion should have arrangements to start an alcohol treatment programme and a responsible support person who can monitor progress, and should not have significant, acute, comorbid conditions or risk factors for severe withdrawal. ${ }^{4}$

Screening to detect problem drinking is recommended in all adult and adolescent patients. ${ }^{5}$ Direct questions about the quantity and frequency of alcohol consumption (to detect hazardous drinkers) and the four-item CAGE questionnaire (to detect dependent drinkers) appear to be the most popular tools to evaluate patterns of alcohol use..$^{6,7}$

The American Society of Addiction Medicines list three immediate goals of detoxification of alcohol and other substances:

- "To provide a safe withdrawal from the drug(s) of dependence and to enable the patient to become drug-free"

- "To provide a withdrawal that is humane and thus protect the patients dignity"

- "To prepare the patient for ongoing treatment of his or her dependence on alcohol or other drugs." ${ }^{8}$

Detoxification manages the signs and symptoms of withdrawal and rehabilitation is intended to help the patient avoid future problems with alcohol. ${ }^{9}$

In practice, in most Irish public healthcare systems, alcohol detoxification services are decoupled from alcohol counselling services and patients usually have to self-refer to counselling services following detoxification. There is usually a waiting list of one to six weeks and some times longer between self-referral and engagement on a counselling programme.

Given the above, the aim of this study was to assess the effectiveness of outpatient alcohol detoxifications as is currently organised in an Irish public psychiatric hospital.

\section{Methodology}

Forty patients who presented to the assessment unit of St Brendan's Hospital with symptoms of ADS and commenced daily outpatient detoxification between the August 1, 2004 and July 31, 2005 were selected for the study.

Names of eligible patients were identified from the outpatient daily records book. Their outpatient charts were examined retrospectively to assess parameters relevant to the objectives of the study, including demographic and clinical data, number of days attended, and requirements for further detoxification. Eight of these patients were excluded from the study due to incomplete outpatient records on them.

For patients who were known to psychiatric sector services, follow up was made with the relevant sector headquarters to determine if the patients regularly attended the service. The results were compiled and analysed using descriptive statistics.

\section{Results}

Forty patients underwent outpatient alcohol detoxification in one year and complete records were available for 32 patients (80\%). Of these 32 patients, 27(85\%) were men and five (15\%) were women. The age range was between 31 and 68 years, with the median age being 43 years. Twenty patients had fixed addresses in the hospital catchment areas, eight patients had no fixed address whilst the remaining four patients had addresses outside the catchment areas as shown in percentages in Figure 1.

\section{Figure 1: Distribution of patients by address of residence}

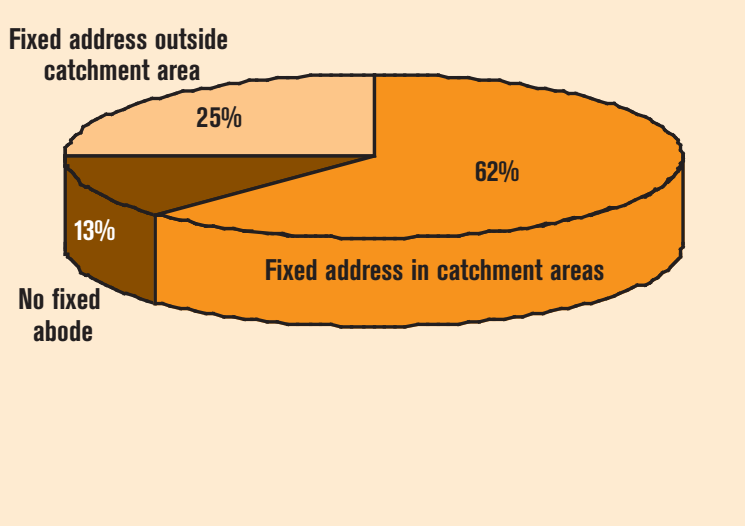

Figure 2: Proportion of patients completing various phases of outpatient detoxification

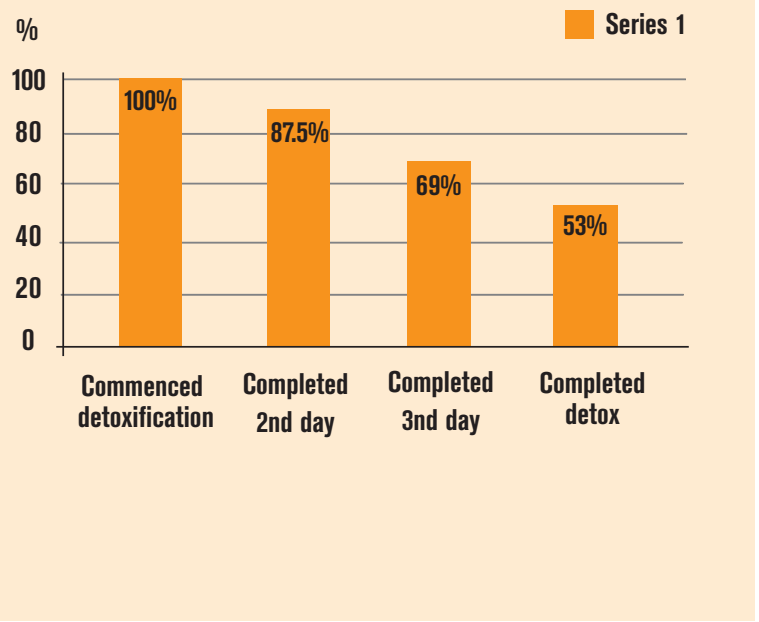

Seven patients (22\%) presented with a co-morbid psychiatric condition including depression (four patients), anxiety disorder (two patients) and personality disorder (one patient). All seven patients were known to psychiatric sector services. For the detoxification, all 32 patients (100\%) consented to and commenced daily outpatient detoxification, 28 patients attended on the second day, 22 patients attended their third day's appointment, and only 17 patients completed the outpatient detoxification as shown in percentages in Figure 2.

Thirteen patients received at least two outpatient detoxifications during the year; of whom seven patients received their second detoxification within two months of the first one as shown in percentages in Figures 3 and 4.

The record of 20 patients $(62.5 \%)$ showed that they had received advice regarding self-referral to counselling services.

\section{Discussion}

Some literature suggests that outpatient detoxification is an effective, safe and low-cost treatment for patients with mild to moderate symptoms of alcohol withdrawal syndrome. ${ }^{10}$ Patients with serious psychiatric involvement (eg. suicidal ideation), concurrent acute illness, or severe alcohol withdrawal syndrome or those who are at risk of developing delirium 
Figure 3: Number of outpatient alcohol detoxifications undertaken per year in the hospital by patients

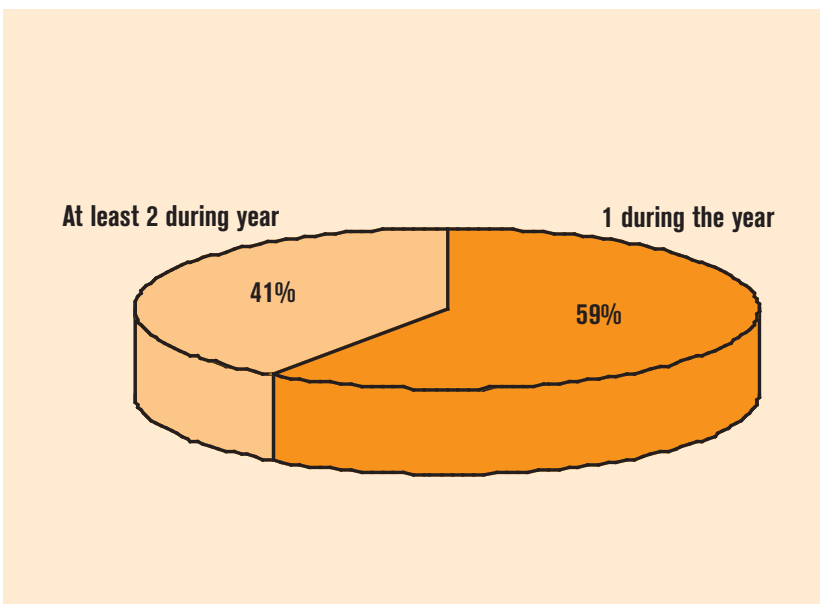

Figure 4: Time scale for second treatment for those receiving multiple detoxifications in the year

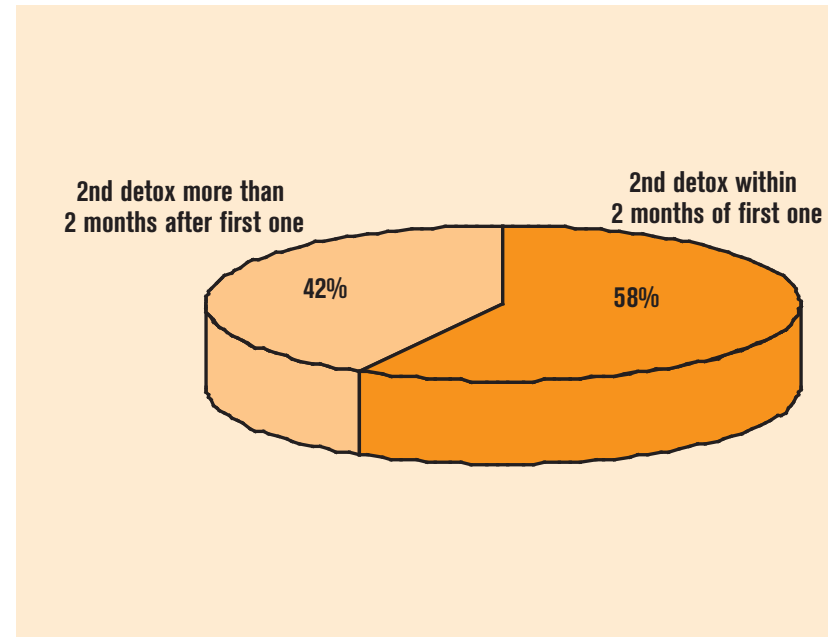

tremens, are best detoxified in an inpatient setting..$^{11-14}$

This study indicates that in one year, at least 40 patients received treatment for alcohol dependency as outpatients in the Assessment Unit of St Brendan's Hospital. A highly significant proportion of these patients received multiple outpatient detoxification during the year, meaning that, the cumulative number of outpatient alcohol detoxification undertaken in the year was very high. This obviously has implications for cost when compared to inpatient detoxification. Consequently, when clinically appropriate, detoxification can be initiated in the ambulatory setting. ${ }^{15}$

The study also revealed that there was a progressive decline in the proportions of patients completing the various phases of the detoxification programme with only as little as $53 \%$ actually completing the whole programme. This raises serious questions about the effectiveness of outpatient treatments.

The study found no significant differences in compliance rates between those with fixed addresses and those without. Further more, the $41 \%$ of the study population receiving at least two detoxifications within the year casts doubts about the success of earlier detoxifications with their supposed follow up counselling. These results are consistent with the results of a study in the US by Finney et al who found that whilst psychosocial treatments show effectiveness in reducing alcohol consumption and maintaining abstinence,
40\%-70\% of patients return to drinking within the year following treatment. ${ }^{16}$ It also compares favourably with the results of studies in Germany by Soyka et a/ which established that only $55 \%$ of sample patients were abstinent and still in outpatient treatment after six months following detoxification. ${ }^{17}$

Of even greater significance is the fact that as many as $58 \%$ of those receiving multiple detoxification actually received the second detoxification within two months of receiving the first one. Several questions arise out of these revelations:

- Do these patients really have the will power and motivation to give up alcohol?

- Is this a benzodiazepine seeking behaviour?

- Should GPs be made responsible for outpatient detoxifications?

- Should outpatient detoxifications and counselling services be provided on the same sites by the addiction treatment services to motivate patients to attend follow-up counselling?

Detoxification does not deal with the psychological issues that cause a person to abuse alcohol. For a successful outcome, people usually have to go through counselling to understand their addiction and change their behaviour. ${ }^{18}$

The proportion of $62.5 \%$ of the study population who were known through records on their charts to have received advice to contact an alcohol counselling service was rather low. This is because, ultimately, the purpose of outpatient detoxification is to facilitate the patient's entry into an alcohol rehabilitation programme. ${ }^{4}$

Finally, with $22 \%$ of the sample population presenting with a co-morbid psychiatric condition, the most common of which was noted to be depression, it is suggestive that alcohol abuse is prevalent amongst this subgroup of people. However, since data on inpatient detoxifications were not considered in the study, it is not possible to draw definitive conclusions about alcohol dependency in people with a comorbid psychiatric problem. Such patients may be candidates for inpatient detoxification. ${ }^{13}$

\section{Conclusion}

The numbers involved in the study were too small to allow for a generalisation of the findings, however, the results are significant when viewed against the background of the increase in ADS in Ireland over the years, and the need to develop appropriate strategies for dealing with the problem.

A high proportion of patients presenting with symptoms of ADS did not complete daily outpatient detoxifications. A high proportion of all patients also underwent multiple outpatient detoxifications during the year. It is possible that the separation between alcohol detoxification and alcohol counselling services contributed to these disappointing results.

Detoxification of eligible patients in local health centres or general practice surgeries which are well resourced at least to initiate counselling of patients during the assessment stage would probably improve outcomes of outpatient treatments.

\section{Acknowledgements}

Special thanks to George Hayes, mental health nurse in St Brendan's Hospital who assisted in retrieving patients' charts. 


\section{Declaration of interest: None}

*Some of the findings of this study were presented as a poster at the Royal College of Psychiatrists Joint Meeting of the Faculty of Addictions and the World Psychiatric Association Annual Residential Conference, Prague, Czech Republic. 4-5 May, 2006.

References

1. Press Release, 22 September 2004, Department of Health and Children, Ireland. Accessed online December 12,2005 at: www.dohc.ie/press/releases/2004/ 20040922.html.

2. O'Connor PG, Schottenfeld RS. Patients with alcohol problems. N Engl J Med 1998; 338: 592-602.

3. National Institute on Alcohol Abuse and Alcoholism. Tenth special report to the U.S. Congress on alcohol and health, 2000. Washington, DC: US Department of Health and Human Services; 2000.

4. Blondell RD. Ambulatory Detoxification of patients with Alcohol Dependence. Am Fam Phys 2005; 71(3)

5. US Preventive Services Task Force. Screening for alcohol misuse. Accessed online December 12,2005 at: www.ahcpr.gov/clinic/uspsdrin.htm.

6. National Institute on Alcohol Abuse and Alcoholism. Helping patients with alcohol problems: a health practitioner's guide. Bethesda, MD: US Dept. of Health and Human Services, National Institute of Health, National Institute on Alcohol Abuse and Alcoholism, 2003, NIH publication no. 03-3769.

7. Gordon AJ et al. Three questions can detect hazardous drinkers. J Fam Pract 2001; 50: 313-20.
8. Kasser C, Geller A, Howell E, Wartenberg A. Detoxification; principles and protocols. American Society of Addiction Medicine. Accessed online December 12, 2005 at: www.asam.org/publ/detoxification.htm.

9. Kenna GA, Mcgeary EJ, Swift RM. Pharmacotherapy, Pharmacogenics, and the Future OF Alcohol Dependence Treatment. Am J Health-Syst Pharm 2004; 61(21): Future OF $2272-2279$.

2272-2279.
10. Hayashida M et al. Comparative effectiveness and cost of inpatient and outpatient detoxification of patients with mild to moderate alcohol withdrawal syndrome. N Engl J Med 1989; 320: 358-65.

11. Mayo-Smith MF. Management of alcohol intoxication and withdrawal. In: Graham AW, Schultz TK, Wilford BB. Principles of addiction medicine. 2nd ed. Chevy Chase, Md.: American Society of Addiction Medicine, 1998: 431-40.

12. Glickman L, Herbsman H. delirium tremens in surgical patients. Surgery $1968 ; 64$ 882-90.

13. Furguson JA, Suelzer CJ, Eckert GJ, Zhou XH, Dittus RS. Risk factors for delirium tremens development. J Gen Intern Med 1996; 11: 410-4.

14. Wojnar M, Bizon Z, Wasilewski D. The role of somatic disorders and physical injury in the development and course of alcohol withdrawal delirium. Alcohol Clin Exp Res 1999; 23: 209-13.

15. Lewis DC. The role of the generalist in the care of the substance-abusing patient. Med Clinic North Am 1997; 81: 831-43.

16. Finney JW, Hahn AC, Moos RH. The effectiveness of inpatient and outpatient treatment for alcohol abuse: the need to focus on mediators and moderators of setting effects. Addiction 1996; 91: 1773-96.

17. Soyka M, Horak M. Ambulatory detoxification of alcohol patients; evaluation of a model project. Gesundheitswesen 2000; 62(1): 15-20

18. Alcohol addiction treatment services in Ireland. Public Information; Dept. of Health and Children, Ireland. Accessed online December 12, 2005 at: www.dohc.ie/public/ information/alcohol_and_drug_treatment_services/alcohol. 